\title{
Research of a hydrodynamic purifying device of exhaust and effluent gases from toxic components under dynamic conditions of their emissions into the atmosphere
}

\author{
Vadim I. Bespalov*, Oksana S. Gurova, Lydmila N. Alekseenko and Anna P. Prokhorova \\ Don State Technical University, 344000 Rostov-on-Don, Russia
}

\begin{abstract}
The article describes the air purifying device of the "OTPO-9" type, which implements the technology of hydrodynamic purification with a foam method where foam was produced by bubbling. The article considers it as the most environmentally efficient and energy-saving device, based on the results of implementation of the methodology of technology selection for purifying exhaust and effluent gases from toxic components of industrial, public and domestic power units up to $1000 \mathrm{~kW}$ developed by the authors. The full-scale laboratory studies of the semiindustrial model of the developed device were carried out by the authors. These studies allowed us to define the nitrogen oxide and sulfur removal efficiency depending on the electric voltage, the shape of the device's side wall in layout, the submersion of inlet and outlet pipe side holes, the ratio of the anode total area to the cathode one. Moreover, the authors have revealed the device scope of application and its advantages. The article states that the proposed device provides environmental safety of the implementation process in comparison with the use of chemical alkaline solutions, which are dangerous for human health and environment.
\end{abstract}

\section{Introduction (Topicality)}

Environmental safety in the development dynamics of built-up areas is related to the creation of comfortable standards of living and protection of human health. However, the need to solve a complex of tasks dealing with dynamics of air pollution control becomes more topical every year. This is due to the factors of material and physical pollution in the current context of urban development which are the most concentrated in the air basin of their territories $[1,2,3]$. The toxic components (nitrogen oxides, carbon monoxide, sulfur oxides, benz(a)pyrene, particles of ash and sootflakes) of exhaust and effluent gases of power units of plants, as well as public and domestic power units have a particular negative effect [4].

The emission level of the toxic components of exhaust and effluent gases of power units of plants, as well as public and domestic power units in the built-up areas would

\footnotetext{
* Corresponding author: okgurova@yandex.ru
} 
significantly exceed the standard values (MAC) without the organization of highly efficient and energy-saving means of purification.

At present, there are many technical solutions (methods, means and devices) providing a dynamic implementation of the purification process of exhaust and effluent gases from toxic components, the operation principle of which is based on various physical mechanisms. Their design is characterized by a different level of complexity, the operating conditions of which are also different $[5,6]$. However, in a number of real situations, several different variants of technical solutions can be applied, providing the same value of purifying efficiency.

For this purpose, we have previously conducted scientific research on the development of evaluation method and applied environmentally and energy efficient technologies for exhaust and effluent gases purification from toxic components to reduce air pollution in urban areas $[7,8,9]$. It allows to solve problem of efficiency and energy saving of the purification process based on the control of its parameters under dynamic conditions of implementation.

\section{Research objective}

The application of the methodology developed by us for the selection of environmentally and energy-efficient technologies for the exhaust and effluent gases purification from toxic components in the conditions of industrial, public and domestic power units operation up to $1000 \mathrm{~kW}$ allows to reveal that air purification device of the type "OtpO-9" [10] is the most environmentally and energy-efficient one, among the modern systems with the maximum values of purification efficiency and energy saving. It presents the technology of hydrodynamic purification with a foam method where foam is produced by bubbling, which was previously used for air purification from fine dust. However, the behaviour and the values of the main operating characteristics of the proposed purification device under dynamic conditions of the emission of toxic components have not been fully investigated and require further development.

\section{Theoretical framework}

We carried out full-scale laboratory studies of the semi-industrial model of the device of the "OTPO-9" type (figure) for the hydrodynamic purification of effluent gases from toxic components (nitrogen oxide and sulfur dioxide) by a foam method where foam was produced by bubbling. It consists of the following main elements (figure): anode 1 and cathode 2 parts of the case; covers 3,4; a flange 5; a membrane 6; electrodes 7; a baffle 8; a whirler 9; inlet 10 and outlet 11 pipes; a drain pipe 12; middle level 13 controller. The dimensions of the device are:

- maximum in longitudinal - $1200 \mathrm{~mm}$;

- maximum in the transversal axis $-750 \mathrm{~mm}$;

- minimum in the transversal axis - $350 \mathrm{~mm}$;

- total height of the device - $1000 \mathrm{~mm}$;

- height of the cone part - $450 \mathrm{~mm}$;

- diameters of inlet and outlet air pipes - $200 \mathrm{~mm}$.

The preliminary research stage involved pre-starting procedure of a model of the device "OTPO-9" type for operating at a laboratory bench. Before loading the effluent gas to device from generator we flooded it so that water level covered side holes of the pipes 10 and 11 not more than half. Each part of the 1st and 2nd device's case was connected to the opposite poles of the DC source (part 1 to "+", part 2 to "-"). 


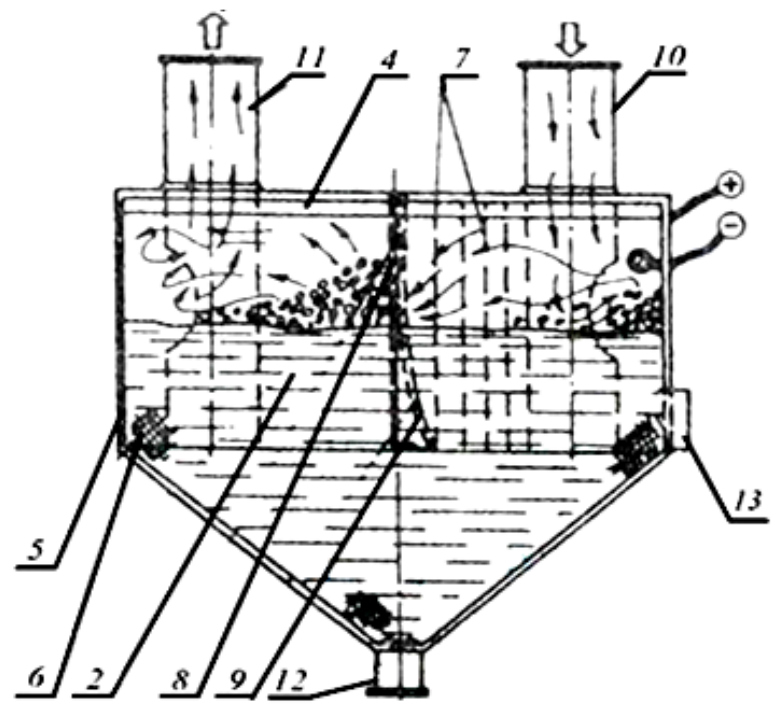

a)

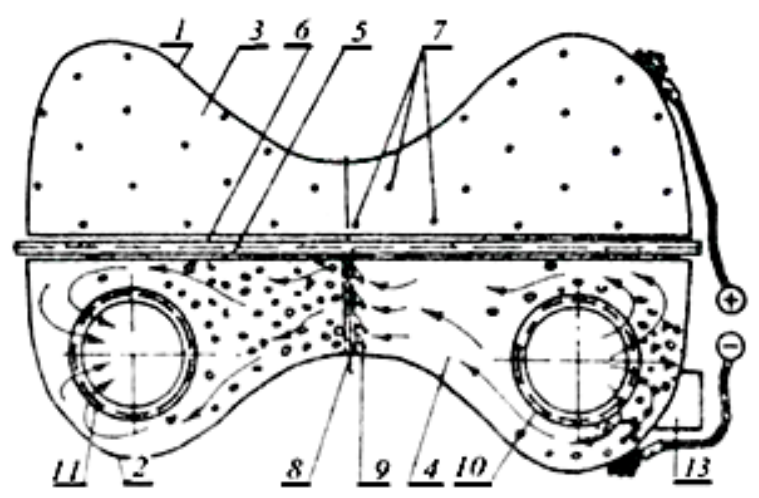

b)

Fig. 1. Effluent gases purifying device of the "OTPO-9". Type a - a side view; $b$ - a top view; 1 - an anod part of the case; 2 - a cathod part of the case; 3, 4-covers; 5 - a flange; 6-a membrane; 7 - electrodes; 8 - a baffle; 9 - a whirler; 10 - an inlet pipe; 11 - an outlet pipe; 12 - a drain pipe; 13 - middle level controller.

As a result, the cover 3 and the electrodes 7 acquire a positive charge, and the cover 4 , the baffle 8 with the whirler 9 , the pipes 10 and 11 the negative charge. Water electrolysis after the connection to the supply source causes the chemical disequilibrium. With that, the anions $[\mathrm{OH}]^{-}$move to the anode $(+)$, and cations $[\mathrm{H} 3 \mathrm{O}]+$ go to the cathode $(-)$. Coming close to the water-permeable membrane 6 , the ions of the corresponding signs are concentrated at the surface of the membrane $6\left([\mathrm{OH}]^{-}\right.$from the side of the cathode, and $[\mathrm{H} 3 \mathrm{O}]+$ from the anode side). In the process, the rate of water activation change (acidity) in a strict limited extent were regulated by voltage and current magnitude. The activation rate (the charge density distribution in liquid) depends on the electrode area and water permeability grade of the membrane material 6 . Thus, the cations $[\mathrm{H} 3 \mathrm{O}]+$ are concentrated 
in the fluid volume between the anode part 1 of the device's case and the membrane 6 , forming an acidic medium with $\mathrm{pH}=5.5-6.0$. And the anions $[\mathrm{OH}]^{-}$are concentrated in the fluid volume between the cathode part 2 of case and the membrane 6 , forming an alkaline medium with $\mathrm{pH}=8.0-8.5$.

The study of the dynamics of the purification process in a device such as "OTPO-9" allowed us to conclude the following. The exhaust gases enter the inlet pipe 10, hit the surface of the fluid, change the direction of their movement, pass through the static whirler 9 and the baffles 8 , flow around the outlet pipe 11 and through its side hole partially free from nitrogen oxide $\mathrm{NO}$ and sulfur dioxide SO2 leave the device. The device's case in the form of a Cassini's oval in layout allowed to ensure a more equal distribution of the charges density in the volume of the fluid by eliminating excess (ballast) zones. The connection of both case parts 1 and 2, the two air pipes 10 and 11 , the baffle 8 , the electrodes 7 to the supply source ensures an equal distribution of charge density (ion concentration) in the fluid volume due to an increase in the surface area of the electrodes. The main effluent gas stream containing toxic components, when entering the device through the inlet pipe 10, hits the surface of the fluid and through the side hole of the inlet pipe 10 goes into the device cavity. Moreover, the effluent gas stream, due to the partial immersion of the side hole of the inlet pipe 10, causes a change in the shape of the fluid surface in the impact area and the surface fluid dropping here. As this process takes place in an alkaline medium characterized by increased wetting and foaming properties, it goes over into the formation of a surface foam layer in which the major part of the toxic components of the effluent gases is retained and captured. The remaining proportion of toxic components with the effluent gas stream enters the zone of action of the static whirler 9 of the baffle 8 , where an intensification of the foaming process is provided. The shape of the Cassini oval contributes to this due to a velocity increase of the contaminated effluent gas stream, its turbulence and, consequently, the increase in the intensity of the interaction of gases with the fluid, thereby forming a foam layer which retains the remaining proportions of toxic components in the stream. As the water is saturated with ions of toxic components in the device, there is its periodically eduction through a drain pipe 12. At the time of draining, the alkaline medium combines with the acidic, forming $\mathrm{pH}$-neutral water, which is environmentally friendly, and which can be recycled by the device. The middle level controller 13 provides fluid supply during operation.

\section{Practical relevance}

The main stage of laboratory studies involved direct measurements. During the main stage of the study, we fixed: the volume of the fluid $-\mathrm{Vl}=0.3 \mathrm{~m}^{3}$; the effluent gas consumption $\mathrm{Qg}=0.25 \mathrm{~m}^{3} / \mathrm{s}$ with their speed at the device inlet $v=4.5 \mathrm{~m} / \mathrm{s}$. At the same time, in each experiment the voltage value of the DC U was varied in the range from 180 to $240 \mathrm{~V}$. Also, the shape of the side wall of the device in layout was changed; the degree of immersion of the side holes of the inlet and outlet pipes $\mathrm{h} / \mathrm{H}$; the ratio of the total area of the anode to the total area of the cathode. Under dynamic conditions for the implementation of the purification process in a device such as "OTPO-9", the measured resultant parameter is the efficiency of effluent gas purification, respectively, from nitrogen oxide $\mathrm{NO}$ and sulfur dioxide $\mathrm{SO}_{2}$. The results of the measurements are shown in Table. 1 and 2, where the numerator is the values of the efficiency of effluent gas purification from nitrogen oxide NO, and the denominator - from sulfur dioxide $\mathrm{SO}_{2}$. 
Table 1. Ratio of the efficiency of effluent gas purification from toxic components to the $\mathrm{pH}$ of the medium and the shape of the side wall of the device in layout, (\%)

\begin{tabular}{|c|c|c|c|c|c|c|}
\hline $\begin{array}{c}\text { The shape of } \\
\text { the side wall of } \\
\text { the device in } \\
\text { layout }\end{array}$ & \multicolumn{7}{|c|}{ pH of alkaline medium } \\
\cline { 2 - 7 } & $\mathbf{7 , 0}$ & $\mathbf{7 , 5}$ & $\mathbf{8 , 0}$ & $\mathbf{8 , 5}$ & $\mathbf{9 , 0}$ & $\mathbf{9 , 5}$ \\
\hline Square & $78,2 / 82,5$ & $79,1 / 83,5$ & $81,0 / 85,9$ & $81,1 / 86,1$ & $81,1 / 86,2$ & $81,2 / 86,3$ \\
\hline Circular & $78,8 / 83,1$ & $80,0 / 85,5$ & $82,8 / 87,1$ & $82,7 / 87,1$ & $82,8 / 87,3$ & $82,8 / 87,4$ \\
\hline Elliptical & $79,3 / 83,9$ & $81,3 / 86,3$ & $84,1 / 89,1$ & $84,2 / 89,4$ & $84,2 / 89,4$ & $84,1 / 89,2$ \\
\hline $\begin{array}{c}\text { In the shape of } \\
\text { Cassini's oval }\end{array}$ & $80,2 / 83,1$ & $82,9 / 86,4$ & $85,6 / 90,3$ & $85,7 / 90,6$ & $85,7 / 90,5$ & $85,8 / 90,7$ \\
\hline
\end{tabular}

Table 2. Ratio of the efficiency of effluent gas purification from toxic components to the total area of the anode and cathode and the existence of statistical whirling, (\%)

\begin{tabular}{|c|c|c|c|c|}
\hline \multirow{2}{*}{$\begin{array}{c}\text { Existence of } \\
\text { statistical } \\
\text { whirling }\end{array}$} & $\begin{array}{c}\text { Degree of } \\
\text { submergence of the } \\
\text { side holes of the } \\
\text { inlet and outlet air } \\
\text { pipes h/H }\end{array}$ & \multicolumn{3}{|c|}{$\begin{array}{c}\text { Ratio of the total area of the anode to the total } \\
\text { area of the cathode }\end{array}$} \\
\cline { 3 - 5 } & 1,0 & $\mathbf{0 , 5}$ & $\mathbf{1 , 0}$ & $\mathbf{1 , 5}$ \\
\hline \multirow{4}{*}{ No } & 0,75 & $60,6 / 64,7$ & $62,8 / 66,5$ & $57,3 / 61,1$ \\
\cline { 2 - 5 } & 0,50 & $75,4 / 79,1$ & $77,7 / 81,0$ & $73,1 / 76,9$ \\
\cline { 2 - 5 } & 0,25 & $83,3 / 87,9$ & $85,8 / 90,7$ & $83,6 / 88,1$ \\
\cline { 2 - 5 } & 0 & $82,3 / 86,9$ & $84,6 / 89,1$ & $80,4 / 84,2$ \\
\cline { 2 - 5 } & & $80,1 / 83,8$ & $81,6 / 85,5$ & $78,3 / 81,5$ \\
\hline
\end{tabular}

\section{Conclusions}

As a result of the full-scale laboratory studies of a semi-industrial model of the «OTPO-9» device for hydrodynamic purifying of effluent gases from toxic components (nitrogen oxide and sulfur dioxide) by a foam method, when foam is produced by bubbling, it can be concluded that the purification efficiency reaches: $85.8 \%$ for nitrogen oxide and $90.7 \%$ for sulfur dioxide $\mathrm{SO} 2$, which is approximately $7.5 \%$ more than for similar bubbling purifying devices.

Table 1 shows that at a $\mathrm{pH}$ of more than 8.0, the purifying efficiency is stabilized, however, achieving $\mathrm{pH}=9.0$ is associated with significant energy consumption. Therefore, the optimum $\mathrm{pH}$ range can be considered 8-9. Moreover, the proposed device provides environmental safety of the process being realized in comparison with the use of chemical alkaline solutions, which are dangerous for human health and the environment.

The scope of the proposed device application and the appropriate purification technology can also be extended to solid and liquid pollutants.

The advantages of the «OTPO-9» device are that it provides: relatively high efficiencies for the toxic components contained in the exhaust and effluent gases; a sufficiently wide range of applications for various types of pollutants; sufficiently high degree of industrial and technological safety. 


\section{References}

2. V.I. Bespalov, O.S. Gurova, N.S. Samarskaya, O.N. Paramonova, Inzhenernyy vestnik Dona, 4, 286 (2013)

3. V.I. Bespalov, O.S. Gurova, N.S. Samarskaya, Biosciences, Biotechnology Research Asia, India, 12, 1587 (2015)

4. V.I. Bespalov, L.Z. Ganicheva, N.V. Yudina, Biosciences, Biotechnology Research Asia, India, 11, 27 (2014)

5. V.I. Bespalov, O.S. Gurova, N.S. Samarskaya, Journal of Scientific Review, 9, 710 (2014)

6. V.I. Bespalov, E.P. Lysova, Journal of Scientific Review, Moscow, 7, 914 (2014)

7. N.S. Samarskaya, T.L. Pirozhnikova, E.P. Lysova, Patent for utility model, $154 \mathbf{4 6 8}$ (2015)

7. V.I. Bespalov, Proceedings of the North Caucasian Scientific Center of Higher Education. Natural Sciences, 37 (1995)

8. E.P. Lysova, Building - 2013: abstracts of doc. Intern. scientific and practical conf., Rostov-on-Don, 122 (2013)

9. E.P. Lysova, L.A. Lysutina, All-Russian scientific and practical conference devoted to the Year of Environmental Protection and the 65th anniversary of the Ufa State Oil Technical University,Ufa, 98 (2013)

10. V.I. Bespalov, O.S. Gurova, S.V. Meshcheryakov, Processes and Apparatuses for Reducing Air Pollution (Rostov-on-Don, 2015) 